\title{
Mass Analysis in Islands of Stability with Linear Quadrupoles with Added Octopole Fields
}

\author{
Nikolai Konenkov \\ Department of General Physics, Ryazan State Pedagogical University, Ryazan, Russia
}

XianZhen Zhao, Zilan Xiao, and D. J. Douglas

Department of Chemistry, University of British Columbia, Vancouver, British Columbia, Canada

\begin{abstract}
Mass analysis with linear quadrupole mass filters is possible by forming "islands" in the stability diagram with auxiliary quadrupole excitation. In this work, computer simulations are used to calculate stability boundaries, island positions, and peak shapes and ion transmission for mass analysis with linear quadrupole mass filters that have added octopole fields of about 2 to $4 \%$. Rod sets with exact geometries that have quadrupole and octopole fields only in the potential, and round rod sets, with multipoles up to $\mathrm{N}=10$ (the twenty pole term) included in the calculations, show the same stability boundaries, island positions, and peak shapes. With the DC voltage applied to the rods so that the Mathieu parameter $a<0$, conventional mass analysis is possible without the use of an island. With the DC polarity reversed so that $a>0$, the resolution and transmission are poor preventing conventional mass analysis. In principle, mass analysis in an island is possible with operation at either of two tips. Provided the correct island tip is chosen for mass analysis, peak shapes comparable to those with $a>0$ and no excitation are possible, both with $a>0$ and with $a<0$. In the latter case, the use of an island of stability allows mass analysis when the added octopole otherwise prevents conventional mass analysis. (J Am Soc Mass Spectrom 2007, 18, 826-834) (C) 2007 American Society for Mass Spectrometry
\end{abstract}

$\mathrm{L}$ inear quadrupole ion traps are finding increasing use in mass spectrometry [1]. Ions can be stored for tandem mass spectrometry $[2,3]$ or confined and manipulated before injection into other mass analyzers, such as time-of flight [4], Paul trap [5], ion cyclotron resonance [6], or electrostatic ("Orbitrap") [7] analyzers.

As with three-dimensional Paul traps [8], the addition of field distortions to a linear quadrupole ion trap can improve MS/MS efficiency [9] or give faster ejection of ions at a stability boundary [10]. The field distortions are described mathematically by the addition of higher spatial harmonics or multipole fields to the quadrupole field. Methods to add octopole [9c, 11] or hexapole [12] fields to linear quadrupoles have been described.

In some applications [3], it is desirable that a linear trap used for MS/MS is capable of mass analysis. Various methods of mass analysis with linear quadrupoles have been described. Conventionally, DC and RF potentials can be applied between the rod pairs [13] to

Published online March 2, 2007

Address reprint requests to Dr. D. J. Douglas, Department of Chemistry, University of British Columbia, 2036 Main Mall, Vancouver, BC, V6T 1Z1, Canada. E-mail: douglas@chem.ubc.ca place ions to be mass analyzed at a tip of a stability region to produce a mass filter. The addition of higher multipoles to the field has, in the past, been expected to degrade the performance of a linear quadrupole operated as a mass filter in this way [14]. Nevertheless, it has been found that linear quadrupoles with added octopole [15] and hexapole fields [12a] can in fact be operated as mass filters, provided the DC voltage is applied to the electrodes with the correct magnitude and polarity. Ions can also be mass analyzed in a linear quadrupole by radial ejection through slots in the rods [2]. Alternatively, axial ejection can be used for mass analysis with a linear quadrupole, either with or without trapping of ions. Ions within the quadrupole are excited by dipole or quadrupole excitation, gain sufficient kinetic energy to overcome a potential barrier at the quadrupole exit, and are ejected $[3,16]$. Preliminary experiments show that this method can be used for mass analysis with a linear quadrupole that has an added octopole field [17].

For operation as mass filters, linear quadrupoles can also be operated with "islands of stability" formed by parametric excitation of ions produced by excitation with auxiliary quadrupole RF or by amplitude modulation of the RF voltage [19]. In a mass filter with a pure quadrupole field, the potential, $V(x, y, t)$, is 


$$
V(x, y, t)=\left(\frac{x^{2}-y^{2}}{r_{0}^{2}}\right) \varphi(t)
$$

where $\mathrm{x}$ and $\mathrm{y}$ are Cartesian coordinates, and $r_{0}$, the field radius, is the distance from the centre to an $x$ or $y$ electrode. With quadrupole excitation, the timedependent potential applied to the electrodes, $\varphi(t)$, is given by

$$
\varphi(t)=U-V_{r f} \cos \left(\Omega t-\phi_{0}\right)-V^{\prime} \cos \omega_{e x} t
$$

where $U$ is the DC potential applied from an electrode to ground, $V_{r f}$ the amplitude of the main RF voltage with angular frequency $\Omega$ applied from pole to ground, $\phi_{0}$ is the initial RF phase, and $V^{\prime}$ is the amplitude of the quadrupole excitation voltage with angular frequency $\omega_{e x}$. With no damping, motion of an ion in the potential of eqs 1 and 2 is determined by

$$
\begin{aligned}
& \frac{d^{2} x}{d \xi^{2}}+\left(a-2 q \cos \left[2\left(\xi-\xi_{0}\right)\right]-2 q^{\prime} \cos 2 v \xi\right) x=0 \\
& \frac{d^{2} y}{d \xi^{2}}-\left(a-2 q \cos \left[2\left(\xi-\xi_{0}\right)\right]-2 q^{\prime} \cos 2 v \xi\right) y=0
\end{aligned}
$$

where the dimensionless parameters are defined as:

$$
\begin{aligned}
& \xi=\frac{\Omega t}{2} ; \xi_{0}=\frac{\phi_{0}}{2} \\
& a=\frac{8 e z U}{m \Omega^{2} r_{0}{ }^{2}} ; q=\frac{4 e z V_{r f}}{m \Omega^{2} r_{0}^{2}} \\
& v=\frac{\omega_{e x}}{\Omega} ; q^{\prime}=\frac{4 e z V^{\prime}}{m \Omega^{2} r_{0}{ }^{2}}=q \frac{V^{\prime}}{V_{r f}}
\end{aligned}
$$

and $m$ is the ion mass, $e$ is the electron charge, and $z$ is the number of charges on the ion.

The angular frequencies of ion oscillation in a quadrupole field, $\omega_{u}$, are given by

$$
\omega_{u}=\left(2 n+\beta_{u}\right) \frac{\Omega}{2}
$$

where $u$ is $x$ or $y, \beta_{\mathrm{u}}$ is a function of the $a$ and $q$ parameters, and $\mathrm{n}=0, \pm 1, \pm 2 \ldots$. With quadrupole excitation, resonances are excited when

$$
\omega_{e x}=\left|l+\beta_{u}\right| \frac{\Omega}{K}
$$

where $K=1,2,3 \ldots$ [20] and $l=0, \pm 1, \pm 2, \pm 3 \ldots$.

If $v=\frac{Q}{P}$ where $Q$ and $P$ are integers, the $\beta$ values of the quadrupole resonances are determined by

$$
\frac{Q}{P}=\frac{\left|l+\beta_{u}\right|}{K}
$$

The stability diagram forms $P-1$ relatively strong resonance lines for the $\mathrm{x}$ and $\mathrm{y}$ motions [18c]. Near the tip of the stability diagram, where $\beta_{x} \approx 1$ and $\beta_{y} \approx 0$, when $v<1$, the strongest resonances occur for the $\mathrm{x}$ motion with $\beta_{x}=Q / P(l=0$ and $K=1$ in eq 8$)$ and for the y motion with $\beta_{y}=1-\frac{Q}{P}(l=-1$ and $K=1$ in eq 8). The stability diagram forms bands of instability along iso- $\beta$ lines, with $\beta_{u}$ values given by eq 8 . Islands of stability are formed between the bands of instability $[18,19]$. Ions of a particular mass to charge ratio $(\mathrm{m} / \mathrm{z})$ can be mass analyzed by adjusting the applied RF and DC voltages to place ions at the tip of an island. Ions of other mass to charge ratios lie outside the stability island and are not transmitted. A mass scan is performed by changing the RF to DC voltages with a constant ratio $U / V_{r f}$ to bring ions of different $m / z$ ratios to the tip of the stability island. Provided the excitation conditions $\left(v, q^{\prime}\right)$ are correctly chosen, a scan line can be produced that passes through the island with the greatest $a, q$ parameters without intersecting other islands of stability. As with a conventional mass filter, the resolution can be adjusted by changing the ratio $U / V_{r f}$. The resolution can be adjusted by changing the ratio $U / V_{r f}$ during a scan to produce the desired peak width (e.g., unit resolution) over the mass range. However the lowest resolution possible will be determined by the width of the stability island $(q / \Delta q \approx 90$ with $v=9 / 10$ and $\left.q^{\prime}=0.020\right)$.

In principle, islands of stability provide an alternate method for mass analysis with linear quadrupoles with added multipoles. The use of an island of stability may allow operation of the rod set as a mass filter under conditions where the added multipoles otherwise compromise mass analysis. The potential in a quadrupole with an added octopole field and no other multipoles, is given by

$$
V(x, y, t)=\left[A_{2}\left(\frac{x^{2}-y^{2}}{r_{0}^{2}}\right)+A_{4}\left(\frac{x^{4}-6 x^{2} y^{2}+y^{4}}{r_{0}^{4}}\right)\right] \phi(t)
$$

where $A_{2}$ and $A_{4}$ are the dimensionless amplitudes of the quadrupole and octopole terms respectively. With quadrupole excitation, $\varphi(t)$ is given by eq 2. (For the potential of eq 9 , the distance from the center to an $x$ electrode is approximately $\frac{r_{0}}{\sqrt{A_{2}}}\left(1-\frac{A_{4}}{2 A_{2}^{2}}\right)$ and to a $\mathrm{y}$ electrode approximately $\frac{r_{0}}{\sqrt{A_{2}}}\left(1+\frac{A_{4}}{2 A_{2}^{2}}\right)$. With an added octopole field, and with $U>0$ ( $a>0$ in eq 5$)$, the boundaries of the stability diagram without excitation are like those of a pure quadrupole field, and are well defined, so that conventional mass analysis is possible. Conversely, when $U<0(a<0)$ the stability boundaries 
move outwards relative to the boundaries with a pure quadrupole field, become diffuse, and the resolution and transmission are substantially decreased [15].

Here, computer simulations are used to calculate in more detail the stability boundaries for quadrupoles with $A_{4} / A_{2} \approx 0.020,0.026$, and 0.040 , for the two cases $U>0$ and $U<0$, both for the case where the field contains quadrupole and octopole terms only (eq 9) and also for the case where the field is created by rod sets with round rods and therefore contains higher multipoles [11]. The positions of the stability islands which are of potential use for mass analysis are calculated. With $\mathrm{U}>0$, the stability boundaries are like those of a pure quadrupole field, regardless of the value of $A_{4}$. The island positions and boundaries depend on the amplitude $A_{4}$. With $U<0$, the stability boundaries move outwards and the positions and boundaries of stability islands again depend on the amplitude $A_{4}$. Provided the correct tip of an island is used, mass analysis in a stability island is possible when $U>0$ and, also when $U<0$ where conventional mass analysis without an island is not possible. Comparable peak shapes and resolution are found in both cases, and these are nearly the same as the same quadrupole operated without excitation and $a>0$. Thus the use of an island of stability allows mass analysis under conditions where the added octopole field leads to poor resolution and transmission.

\section{Methods}

In a linear quadrupole with field distortions or added higher multipoles, the potential is given by

$$
V(x, y, t)=\varphi(t) \sum_{N=0}^{\infty} A_{N} \phi_{N}(x, y)
$$

where $A_{N}$ is the dimensionless amplitude of a multipole $\phi_{N}(x, y)$, and $\varphi(t)$ is determined by the time-dependent voltage applied to the electrodes. If the potential contains only quadrupole and octopole terms, $V(x, y, t)$ is given by eq 9. (This potential can be produced by electrodes with exact shapes calculated from $V(x, y)=$ \pm 1 with $V(x, y)$ given by the spatial dependence of eq 9 i.e., $V(x, y, t)$ with $\varphi(t)=1)$. A quadrupole with an added octopole field can be constructed with round rods equally spaced from a central axis at a distance $r_{0}$, and with the radius of the y rods, $R_{y}$, greater than the radius of the $\mathrm{x}$ rods, $R_{x}$ [11]. In this case, the $x$ direction is the direction of the smaller rods, and $A_{4}>0$. This co-ordinate system is used here. The use of round rods contributes additional higher multipoles to the potential [11]. The properties of round rod sets with nominal 2 to $4 \%$ added octopole fields are being investigated experimentally in this lab [9a, 15]. The amplitudes of the multipoles of these round rod sets, calculated as in [21], and the relative rod radii are shown in Table 1. These are referred to as rod sets with "2, 2.6 and $4 \%$ " added octopole fields, although the exact values of $A_{4}$ used are
Table 1. Multipole amplitudes for the round rod sets investigated. In all cases $R_{x}=r_{0}$. The multipoles for rod sets with 2.0 and $2.6 \%$ added octopole fields were calculated including a grounded case of radius $4 r_{0}$.

\begin{tabular}{|c|c|c|c|}
\hline$A_{N}$ & $\begin{array}{c}2 \% \text { octopole } \\
\qquad R_{y} / R_{x} \\
=1.220\end{array}$ & $\begin{array}{c}2.6 \% \text { octopole } \\
R_{y} / R_{x} \\
=1.304\end{array}$ & $\begin{array}{c}4.0 \% \text { octopole } \\
R_{y} / R_{x} \\
=1.516\end{array}$ \\
\hline$A_{2}$ & 0.9994 & 1.001 & 1.006 \\
\hline$A_{4}$ & 0.01957 & 0.02592 & 0.03989 \\
\hline$A_{6}$ & 0.003280 & 0.001191 & -0.002983 \\
\hline$A_{8}$ & 0.0008659 & 0.0009596 & 0.0008572 \\
\hline$A_{10}$ & -0.0002344 & -0.002357 & -0.002322 \\
\hline
\end{tabular}

given in Table 1. Mass analysis with these rod sets is modeled in this study. Unless otherwise stated, multipoles up to $N=10$ were included in all the calculations for the round-rod sets.

When $U>0$ and $a>0$, an island is formed in the upper part of the stability diagram and it is convenient to refer to the "upper" and "lower tips" of the island. When $U<0$ and $a<0$, references to the upper and "lower" tips of the island can be confusing, and we refer to the tips with the greater and lesser magnitudes of $a$.

\section{Peak Shapes and Boundary Calculations}

Stability boundaries and peak shapes were calculated as described previously [12a, 22]. Ion trajectories were run to determine if ions have stable or unstable motion for given values of the $a$ and $q$ parameters. Initial spatial positions were selected from Gaussian distributions in $\mathrm{x}$ and y with standard deviations $\sigma_{x} / r_{0}=0.002$. Initial velocities were selected for an ion of mass $390 \mathrm{Da}$, with a thermal distribution with temperature $\mathrm{T}=300 \mathrm{~K}$. With quadrupole frequency $\mathrm{f}=1.0 \times 10^{6} \mathrm{~Hz}$ and $r_{0}=$ $5 \times 10^{-3} \mathrm{~m}$, this gives a dimensionless velocity dispersion [12a] of $\sigma_{v}=\frac{\sqrt{\frac{2 k_{B} T}{m}}}{\pi r_{0} f}=0.007$ (where $k_{B}$ is Boltzmann's constant). Twenty initial RF phases, $\phi_{0}$, uniformly distributed between 0 and $2 \pi$ were used. The phase of the excitation was fixed (eqs 2 to 4 ). The effects of the phase of the excitation are unknown and remain to be explored. In experiments where the auxiliary excitation was not phase locked to the quadrupole RF voltage, Konenkov et al. [18a] found good agreement between the experimental island positions and the theoretical positions calculated with phase locked excitation, suggesting that the phase of the excitation may not be important. For the calculation of stability boundaries and island positions, $a$ was fixed and $q$ was systematically varied to produce a curve of transmis- 
sion versus $q$. For the boundaries without quadrupole excitation $q^{\prime}$ was set to zero. Values of $a$ and $q$ giving $1 \%$ transmission were taken as stability boundaries. For peak shape calculations, the variation of transmission with $q$ was determined as $a$ and $q$ were varied systematically along a scan line with slope

$$
a / q=2 \lambda=\frac{2 U}{V_{r f}}
$$

In all calculations, ions spent $150 \mathrm{rf}$ cycles in the field, $q$ was varied in step sizes typically of 0.0002 to 0.0004 , and 4000 to 6000 trajectories were run for given $a$ and $q$ values. Reported resolutions, $R_{1 / 2}$, are calculated from peak full widths at half maximum, $q / \Delta q$. All calculations with quadrupole excitation were done for $v=$ $9 / 10$ (as in [18a, c]) and $q^{\prime}=0.020$. The widths of the bands of instability increase as $q^{\prime}$ increases [18a, c]. With $q^{\prime}=0.020$ the bands of instability are sufficiently broad that the scan lines investigated do not pass through any other islands of stability. No fringing fields were included in the calculations.

The calculations give the boundaries of islands or stability diagrams where the transmission is $1 \%$. Because the initial conditions are selected randomly, there is a statistical uncertainty in the calculated transmission. If the number of trajectories run at a point $(a, q)$ is $N$, and the number of ions transmitted is $N_{t}$, the transmission is $N_{t} / N$, and the uncertainty in the transmission is $\sqrt{N_{t}} / N_{t}$. Peaks with low transmission therefore have a "noisy" appearance.

\section{Results and Discussion}

\section{Peak Shapes with Conventional Mass Analysis}

In [15] peak shapes were calculated for a quadrupole with a $2.0 \%$ added octopole field and no other multipoles. For direct comparison to the peaks shapes and stability boundaries with islands calculated below, peak shapes and boundaries without excitation were calculated for round rod sets with 2, 2.6, and $4 \%$ added octopole fields, including all the multipoles up to $N=$ 10 , and with the ion source model described above. Figure 1a shows peak shapes for a round rod set with $2.6 \%$ added octopole field with the positive DC applied to the smaller rods so that $a>0$. The resolutions of the peaks are from 384 to 705 . Figure 1 b shows peak shapes for the same rod set but with the negative DC applied to the smaller rods so $a<0$. For a given transmission the resolution is lower, and there are tails on the high mass sides of the peaks. For example at a transmission of 0.026 the resolution is 238 compared to a resolution of 705 with a transmission of 0.032 in Figure 1a. These results are qualitatively consistent with the experimental and calculated peak shapes of [15]. With $a>0$, peak shape and resolution comparable to those of a quadrupole with no added octopole field, are possible. With the polarity of the DC reversed $(a<0)$, poor peak shape
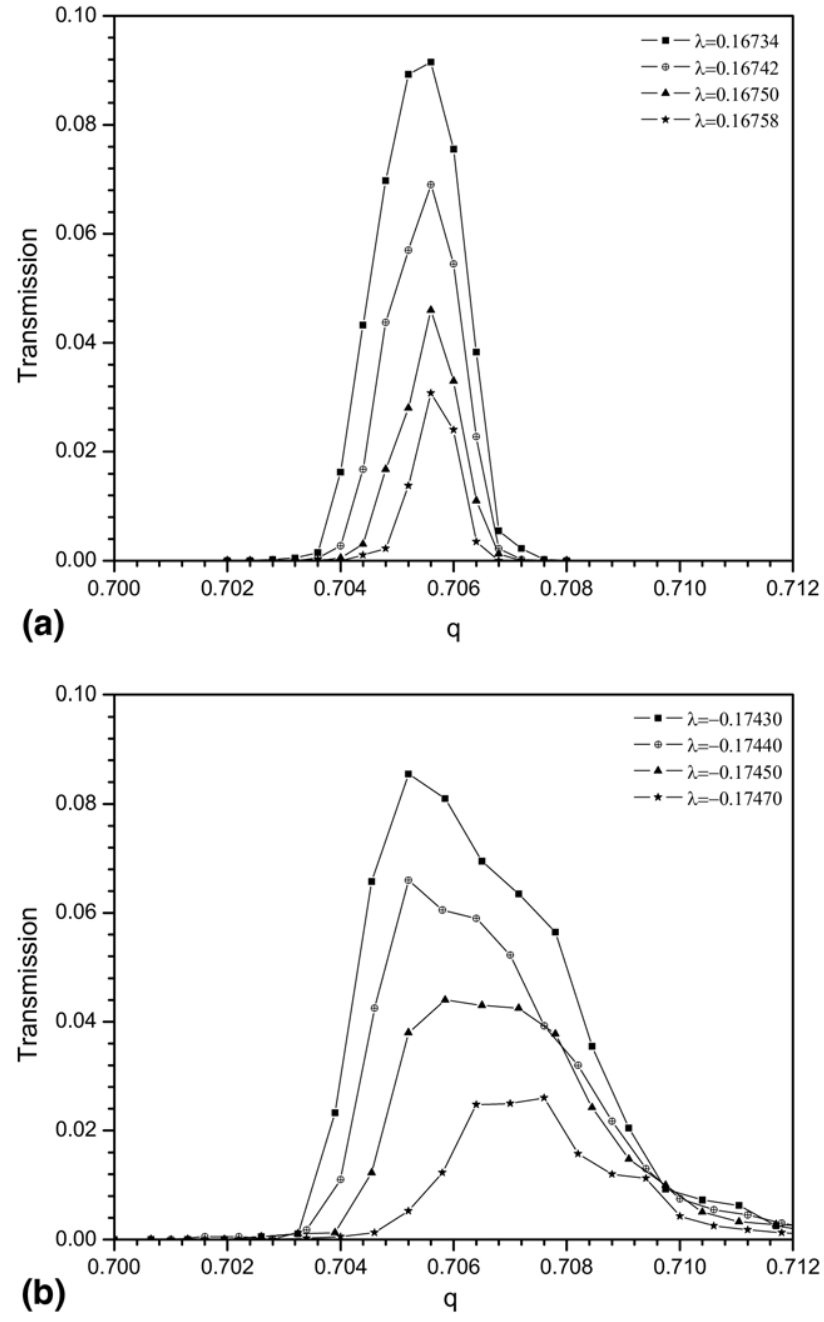

Figure 1. Peak shapes for four values of $\lambda$ for a round-rod set with $2.6 \%$ added octopole field without additional quadrupole excitation $\left(q^{\prime}=0\right)$ with (a) $a>0$ and (b) $a<0$.

and resolution are obtained because the stability boundaries become diffuse [15].

\section{Islands and Stability Boundaries}

The stability boundaries and island positions were compared for rod sets that have quadrupole and octopole terms only [i.e., only $A_{4} \neq 0$ and $A_{2} \neq 0$ (eq 9)] and for round rod sets with $2,2.6$, and $4 \%$ added octopole fields. For example, for the quadrupole with $2.6 \%$ octopole, a quadrupole with same values of $A_{2}$ and $A_{4}$ (Table 1) but with all the other multipole amplitudes, $A_{N}$, set to zero was simulated. These calculations show that the higher multipoles added by the use of round rods do not substantially change the island positions or stability boundaries. This differs from the case of quadrupoles with added hexapole fields, where the higher multipoles cause small shifts of the stability boundaries [12a].

The effect of the polarity of the DC voltage applied to 


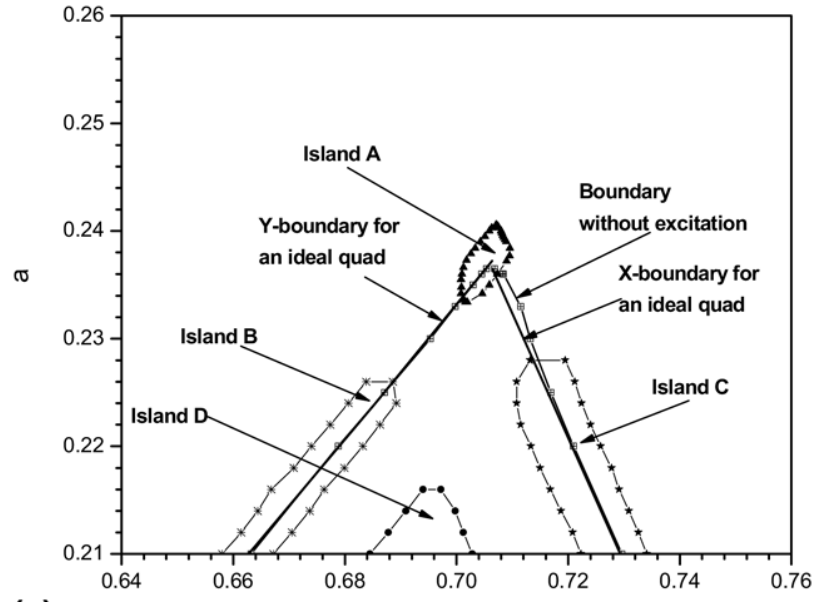

(a)

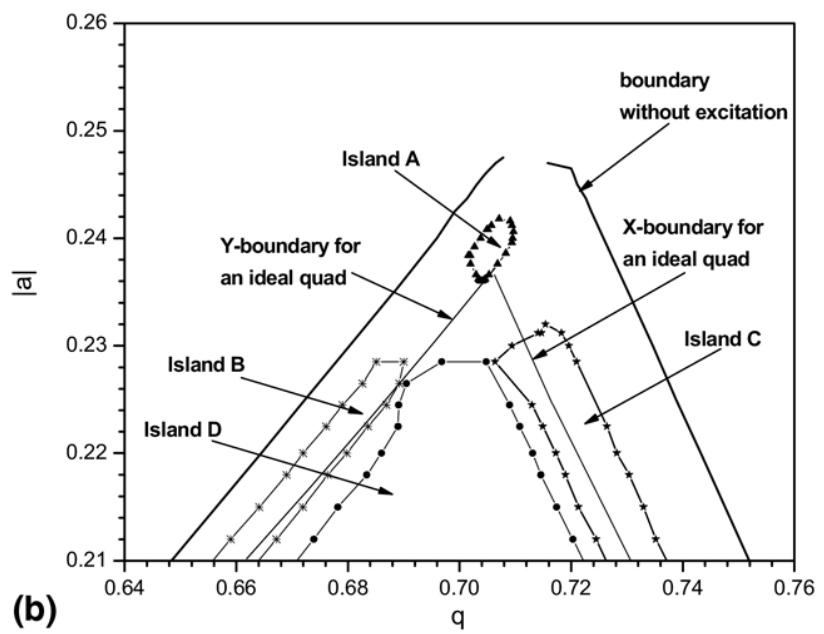

Figure 2. Stability boundaries $\left(q^{\prime}=0.00\right)$ and islands of stability $\left(q^{\prime}=0.02, v=9 / 10\right)$ for a quadrupole with $2.6 \%$ added octopole field with (a) $a>0$ and (b) $a<0$.

the quadrupole electrodes, i.e., the sign of $a$, was investigated for round rod sets with 2, 2.6, and $4 \%$ added octopole fields. Figure 2 shows the calculated stability boundaries and islands of stability for a roundrod set with $2.6 \%$ octopole field. The islands are labeled as in [18a]. With $a>0$, Figure 2a, and without excitation, the $\mathrm{x}$ and $\mathrm{y}$ boundaries of the stability diagram are nearly identical to the boundaries with a pure quadrupole field. In this case the added octopole field does not cause significant changes to the stability diagram. The island boundaries however, differ from those with a pure quadrupole field (see below). In principle, these islands can be used for mass analysis.

Figure $2 \mathrm{~b}$ shows the case with $a<0$. There are significant changes to the islands and stability boundaries. The boundaries without excitation move outwards to greater $a, q$ values and become diffuse. Because the boundaries move outwards, greater values of $\lambda$ are required for mass analysis at a given resolution. The islands A, B, and C have boundaries $(|a|, q)$ similar to those with $a>0$. The boundaries of island D change substantially and move out towards the inner boundaries of islands B and C. Thus the "gaps" between island D and islands B and C become smaller. Because the stability boundaries without excitation move outwards, island A now appears within these boundaries, although it has $|a|, q$ values similar to the case with $a$ $>0$.

For comparison, the stability boundaries and islands of round rod sets with added octopole fields of 2, 2.6, and $4 \%$ are shown in Figure 3. With $a>0$, the stability boundaries without excitation for these rod sets were found to be nearly identical, and like those of a quadrupole with a pure quadrupole field (Figure 2a). With $a$ $<0$, the stability boundaries without excitation depend on the amplitude $A_{4}$. Both with $a>0$ and $a<0$, the boundaries and positions of the islands depend on the amplitude $A_{4}$. The greatest changes with $A_{4}$ occur with $a<0$.

Figure 3a shows islands of stability with $a<0$ for round rod sets with $2 \%$ and $2.6 \%$ added octopole fields. Also shown are stability boundaries without excitation for rod sets with $0,2,2.6$, and $4 \%$ added octopole fields. As $A_{4}$ increases, the $\mathrm{x}$ stability boundary moves outwards. The y stability boundary also moves outwards, but to a lesser extent. Island $\mathrm{A}$ has nearly the same position for values of $A_{4}$ of 2.0 to $4.0 \%$ (see below). Islands $\mathrm{B}$ and $\mathrm{C}$ have similar positions, although the boundaries change slightly with $A_{4}$. The largest changes with $A_{4}$ occur for island $\mathrm{D}$. The right boundary moves outwards with increasing $A_{4}$. With a $4.0 \%$ added octopole field, the boundary between island $\mathrm{D}$ and $\mathrm{C}$ cannot be detected in these calculations and therefore the islands with $4 \%$ added octopole are not shown in Figure 3a. As $A_{4}$ increases, the left boundary of island $\mathrm{D}$, also moves outwards but to a lesser extent.

Figure $3 \mathrm{~b}$ shows the stability islands A both for $a>$ 0 and $a<0$ for quadrupoles with $0.15,2,2.6$, and $4 \%$ added octopole fields. Also shown, by the shaded area within solid lines, is the stability island for a pure quadrupole field. The overlapping area of the stability islands for $\pm a$ is the island with no added octopole field. With $a>0$ the two upper boundaries of island A (the boundaries with greater $a, q$ values nearly coincide with those of a pure quadrupole. The two lower boundaries, labeled (2) and (4) in the figure, which are formed by the resonant excitation band, move away from the island of a pure quadrupole. With $a<0$, the lower boundaries in the figure, which are formed by the resonant excitation band, remain nearly the same as those of a pure quadrupole, and the upper boundaries, labeled (1) and (3), move outwards. The boundaries of island $\mathrm{A}$ for $A_{4}=0.02,0.026$, and 0.04 are very similar. The island for $A_{4}=0.0015$ show boundaries intermediate between those of $A_{4}=0$ and $A_{4}=0.020$.

A scan line with $\lambda=0.17005$ passes through the upper tip (greater $|a|$ ) of island A with $a>0$ and a scan line with $\lambda=0.16765$ passes through the tip with the lesser $|a|$ with a $<0$. These two tips, which correspond to boundaries that are relatively unperturbed 


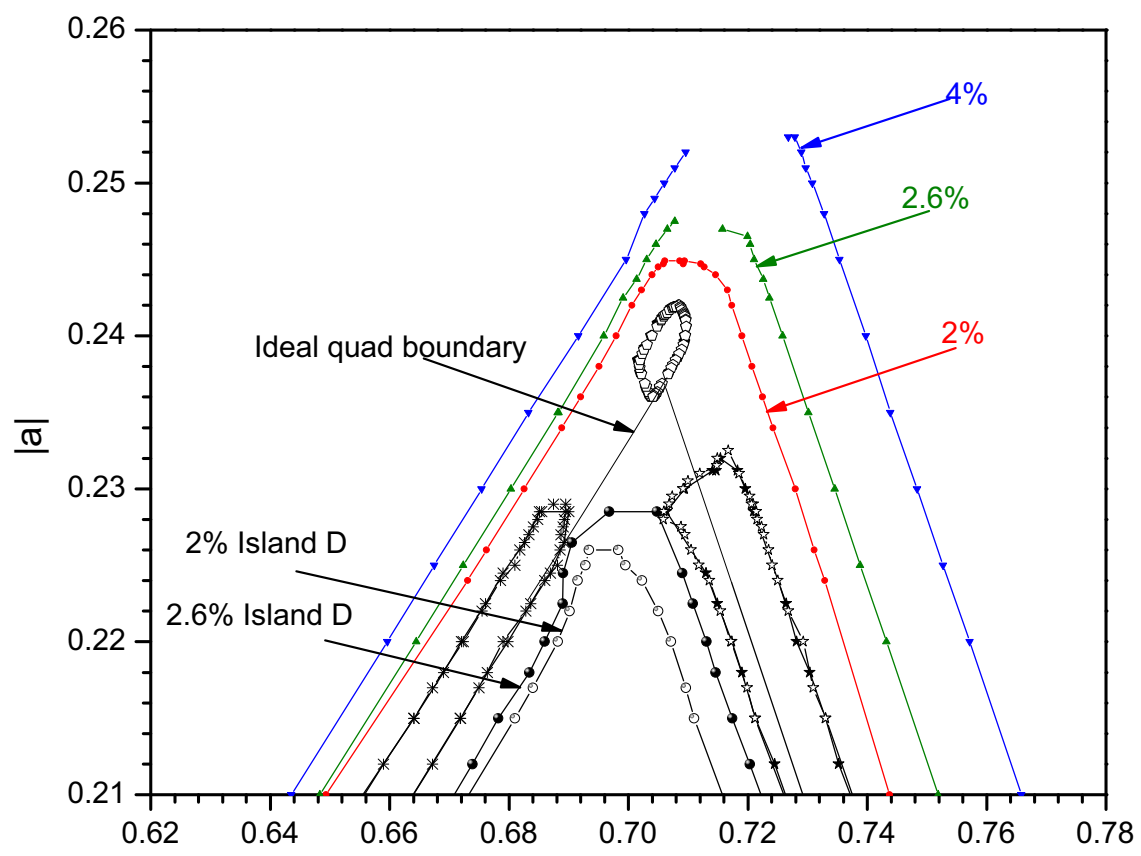

(a)

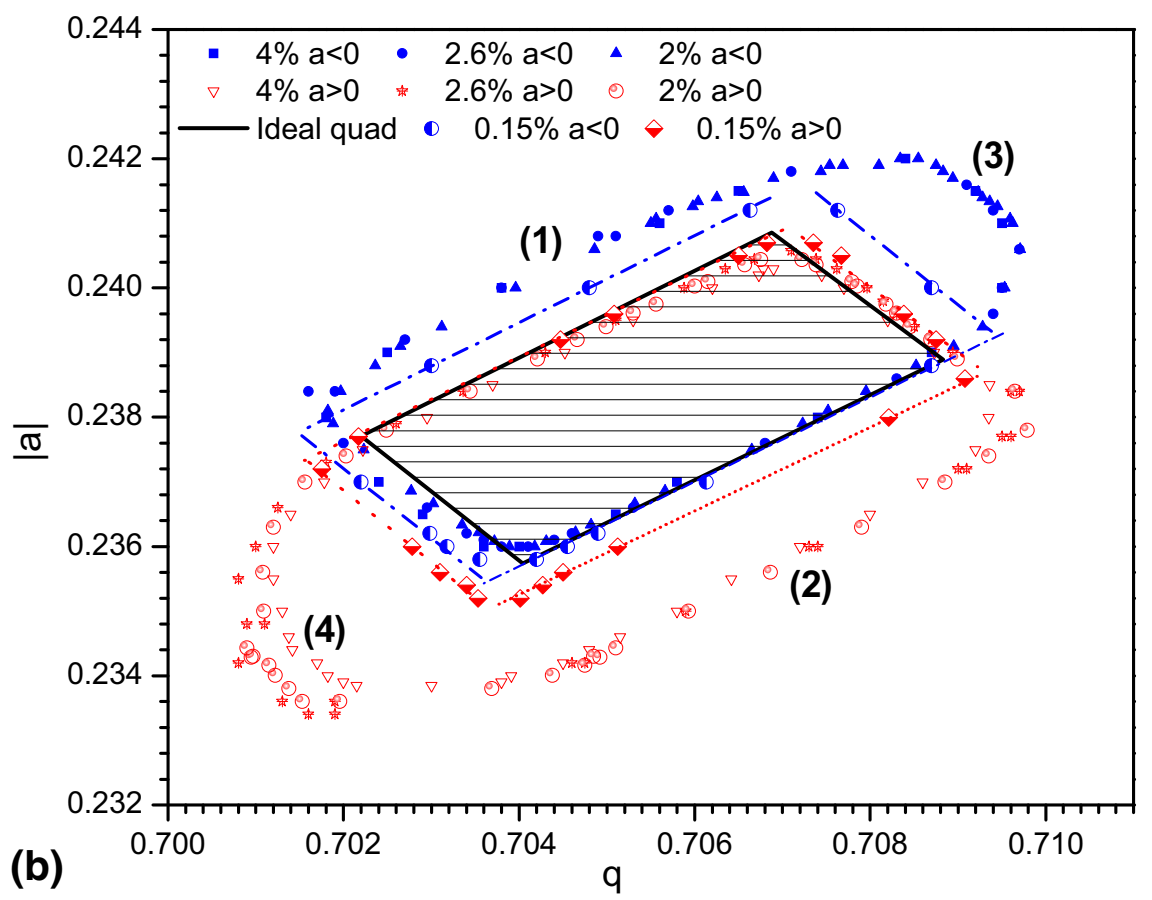

Figure 3. (a) Stability boundaries $\left(q^{\prime}=0.00\right)$ and islands of stability $\left(q^{\prime}=0.020\right.$ and $\left.v=9 / 10\right)$ for quadrupoles with $2,2.6$, and $4 \%$ added octopole fields constructed with round rods, $a<0$. (b) Island A for quadrupoles with 0.0 (ideal quadrupole, solid line boundaries of the shaded area), 0.15, 2, 2.6, and $4 \%$ added octopole fields, with $a>0$ and $a<0$.

by the addition of the octopole field, provide the highest resolution and best peak shapes as described below.

The $\mathrm{x}$ and $\mathrm{y}$ stability boundaries without excitation $\left(q^{\prime}=0\right)$ with $a<0$ (Figure 3a) show unequal shifts for $0.02<A_{4}<0.04$. To investigate this further, the $q$ values of the $\mathrm{x}$ and $\mathrm{y}$ stability boundaries, with $a=0.240$ were calculated for round rod sets with added octopole fields in the range $0<A_{4}<0.040$. The results are shown in Figure 4a. It is seen that the shifts are nonlinear with $A_{4}$ with the y boundary showing greater shifts for $A_{4}<$ 0.01 and the x boundary showing greater shifts for $A_{4}>$ 

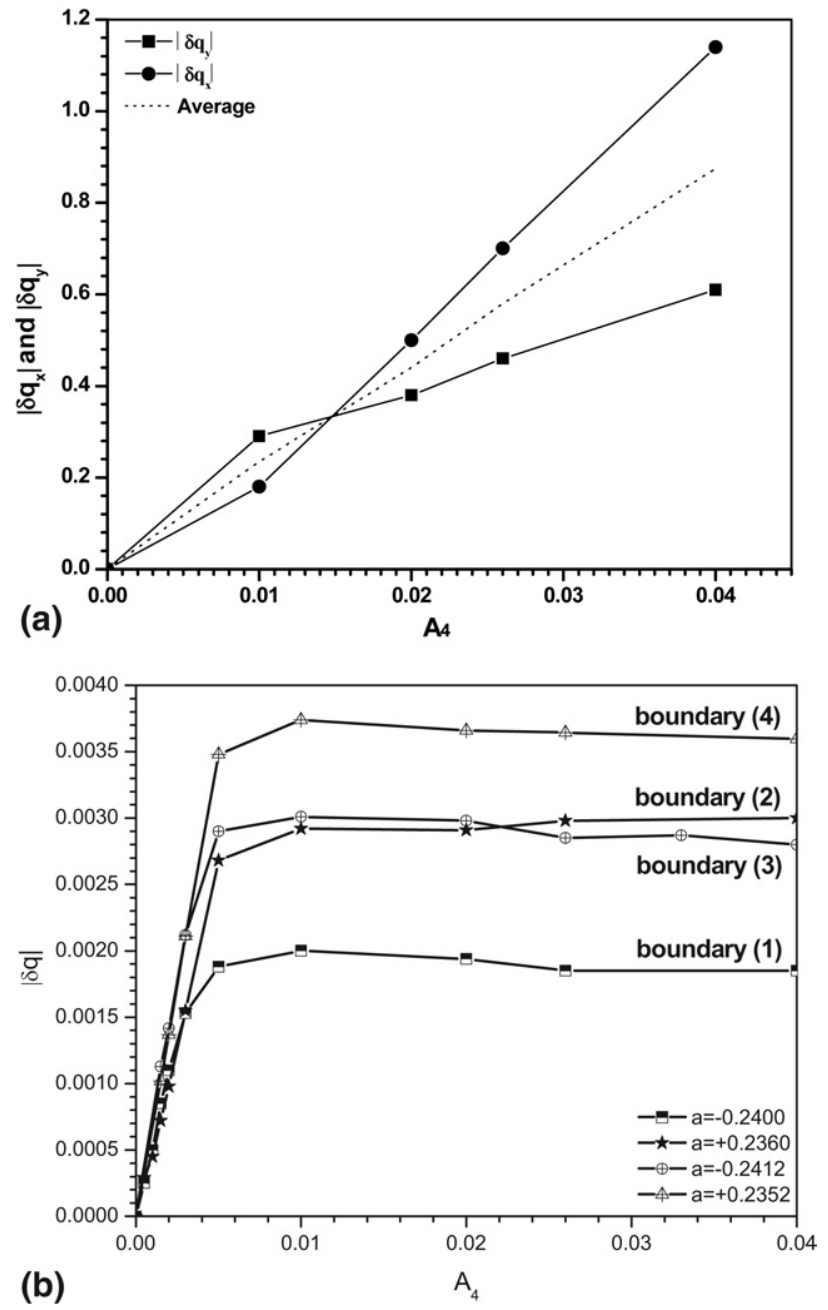

Figure 4. (a) Magnitudes of the shifts of the values of $q$ of the positions of the $x$ and $y$ stability boundaries, from those of an ideal quadrupole, calculated at $a=+0.2400$ versus $A_{4}$ for rod sets constructed with round rods with $a<0$. (b) Shifts of the positions of the stability boundaries (1) to (4) of island A versus $A_{4}$ with both $a>0$ and $a<0$.

0.01 . The average shift is nearly linearly proportional to $A_{4}$ and the data are symmetric about this line.

The island boundaries in Figure $3 \mathrm{~b}$ also show unequal shifts with different values of $A_{4}$ and for $\pm a$. To further investigate how the islands move with $0.0 \leq A_{4}$ $\leq 0.04$, the values of $q$ of the boundary of island A were calculated for $a=+0.2352,+0.2360,-0.2412,-0.2400$, with the results shown in Figure $4 \mathrm{~b}$. Values of $A_{4}<$ 0.005 cause significant shifts in the boundary, while for $0.01 \leq A_{4} \leq 0.04$ any additional shifts are small.

\section{Mass Analysis in Islands of Stability}

Figure 5a shows peak shapes with $a>0$ with mass analysis at the upper tip (greater $|a|$ ) of island A with values of $\lambda$ from 0.16980 to 0.17005 . The peaks have resolutions of 380 to 744 . Approximately doubling the resolution from 380 to 744 causes the transmission to decrease by about a factor of four. These values of $\lambda$ give scan lines which pass outside the conventional stability region (where, at the tip, $\lambda=0.16784$ ). Good peak shape and resolution are obtained. The peaks are free of tails.

Figure $5 \mathrm{~b}$ shows peak shapes with mass analysis at the lower tip (lesser $|a|$ ) of island A with $a>0$. Here, for a given transmission the resolution is relatively poor. For example at a transmission of about $0.04, R_{1 / 2}=275$, whereas with operation at the upper tip $R_{1 / 2}=540$, for the same transmission. When $\lambda$ is decreased from 0.16655 to 0.16645 in an effort to increase the resolution, the nominal resolution decreases from 382 to 293 . As well, the peaks have tails on the high mass side and undesirable structure. Clearly with $a>0$ the upper tip of island $\mathrm{A}$ is superior for mass analysis.

Figure 6a shows peak shapes for mass analysis in island A with $a<0$ at the tip with the greater $|a|$. For a given transmission, the resolution is relatively low (e.g., at a transmission of $0.022, R_{1 / 2}=245$ compared to $R_{1 / 2}=540$ in Figure $5 \mathrm{~b}$ ) and structure appears on the peaks.
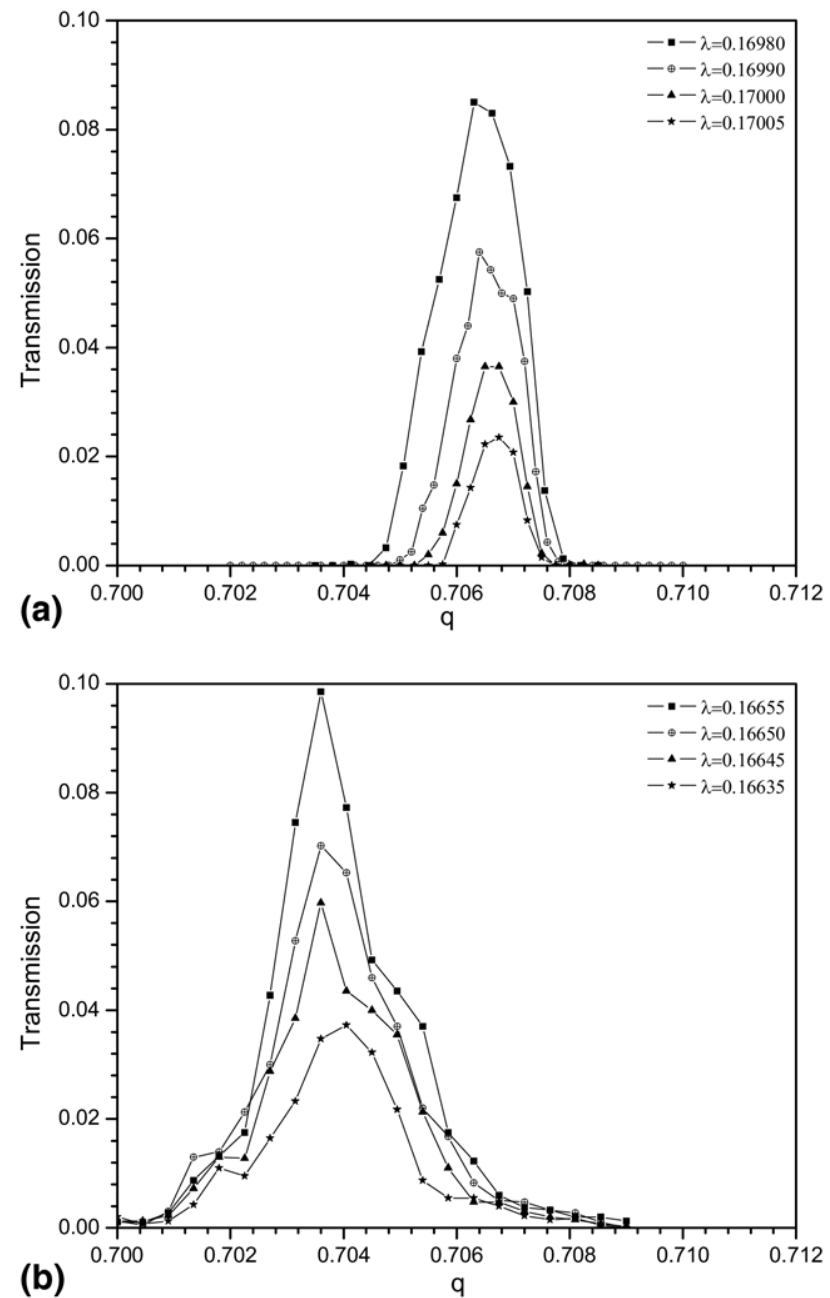

Figure 5. Peak shapes for mass analysis in island A for a rod set with $2.6 \%$ added octopole field and $a>0$ at (a) the upper tip (the tip with greater $|a|$ ) and (b) the lower tip (the tip with lesser $|\mathrm{a}|$ ). 

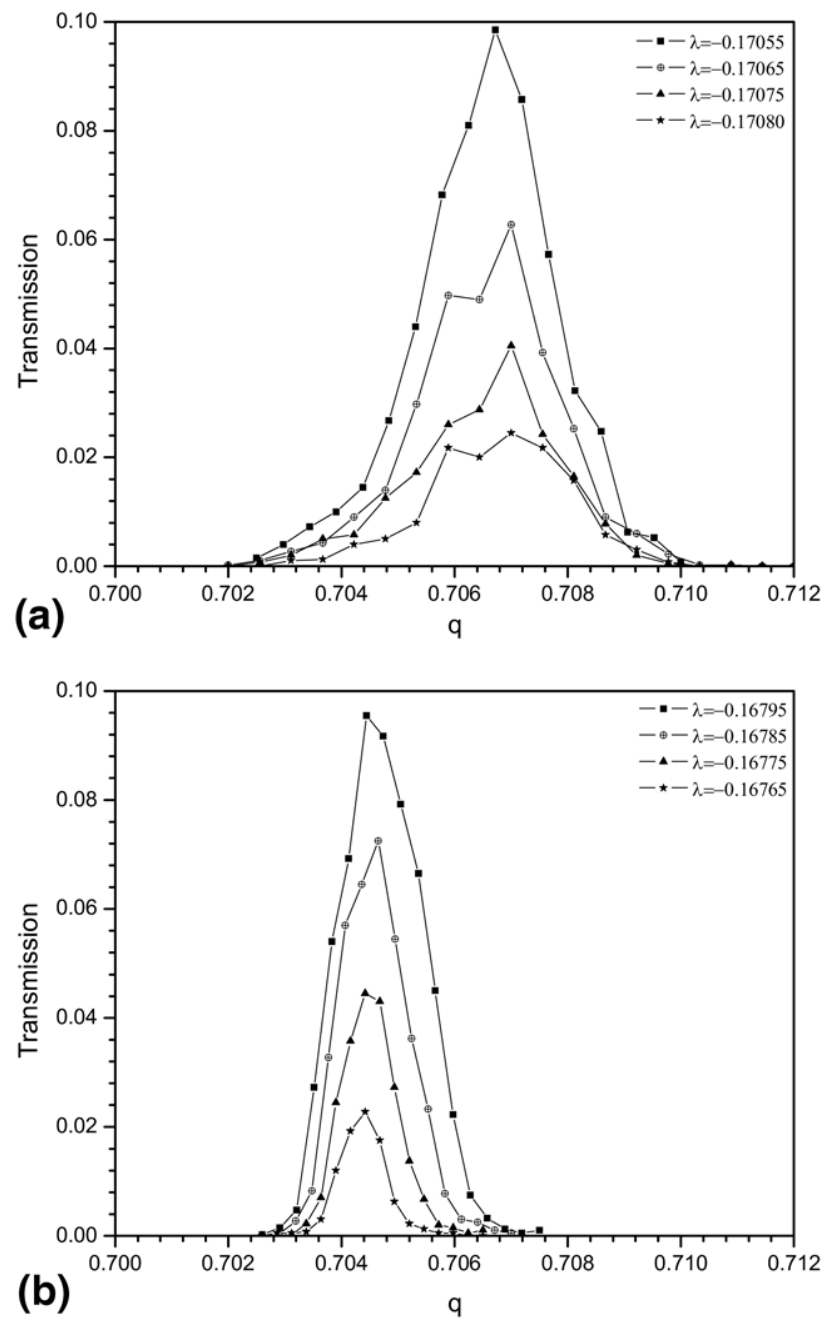

Figure 6. Peak shapes for mass analysis in island A for a rod set with $2.6 \%$ added octopole field and $a<0$ at (a) the tip with the greater $|a|$ and (b) the tip with the lesser $|a|$.

Figure $6 \mathrm{~b}$ shows peak shapes for mass analysis in island A with $a<0$ at the tip with the lesser $|a|$. The peak shape, transmission and resolution are improved compared to operation at the tip with the greater $|a|$. The peaks have resolution from 380 to 767 , are free of structure and do not tail on the high or low mass side. The peaks are comparable to those with conventional mass analysis with a $>0$ and no excitation applied. Thus this island allows mass analysis when the added octopole otherwise prevents mass analysis. Calculated peak shapes and transmission for operation in island $\mathrm{A}$ with quadrupoles with $A_{4}=0.02$ and $A_{4}=0.04$ are very similar (data not shown).

Figure 7 shows peak shapes at $R_{1 / 2} \approx 700$ for (a) a quadrupole with a pure quadrupole field and no excitation, (b) a quadrupole with $2.6 \%$ octopole and round rods operated with $a>0$ at the tip of the stability diagram with no excitation, (c) a quadrupole with $2.6 \%$ octopole and round rods operated with $a>0$ at the tip of island A with the greater $|a|$, and (d) a quadrupole with $2.6 \%$ octopole field and round rods with $a<0$ operated at the tip of island A with the lesser $|a|$. It is seen that with the rod set with $2.6 \%$ added octopole, operation at the preferred tips of island A gives peak shapes and transmissions similar to that of the same quadrupole operated at the tip of the stability island with no excitation. Thus, with $a<0$, the use of the island of stability provides resolution and transmission similar to that with $a>0$. However all the peaks for the quadrupole with $2.6 \%$ added octopole have transmission that is lower than the peak of a pure quadrupole field operated without excitation by a factor of about 25 . (The higher transmission for peak $a$ results in a statistical error lower by about $\times 5$ ). Experiments comparing the transmission versus resolution of a round rod quadrupole with a $2.0 \%$ added octopole field to a conventional rod set constructed with round rods, did not show this large difference in transmission [15]. The rod set with added octopole showed lower transmission than the conventional rod set, but only for resolutions greater than about 1500. The transmission of mass analyzing quadrupoles with added multipoles and the dependence of the transmission on the source characteristics require additional investigation. Preliminary results show that the fringing fields at the entrance to a quadrupole with an added octopole field can increase the transmission by about a factor of ten to give transmission comparable to that to a conventional round rod quadrupole [23].

The results here show that the best resolution and peak shape are obtained with operation at the peak of island A with the greater $|a|$ with $a>0$ and the lesser $|a|$ with $a<0$. Similar results were obtained for

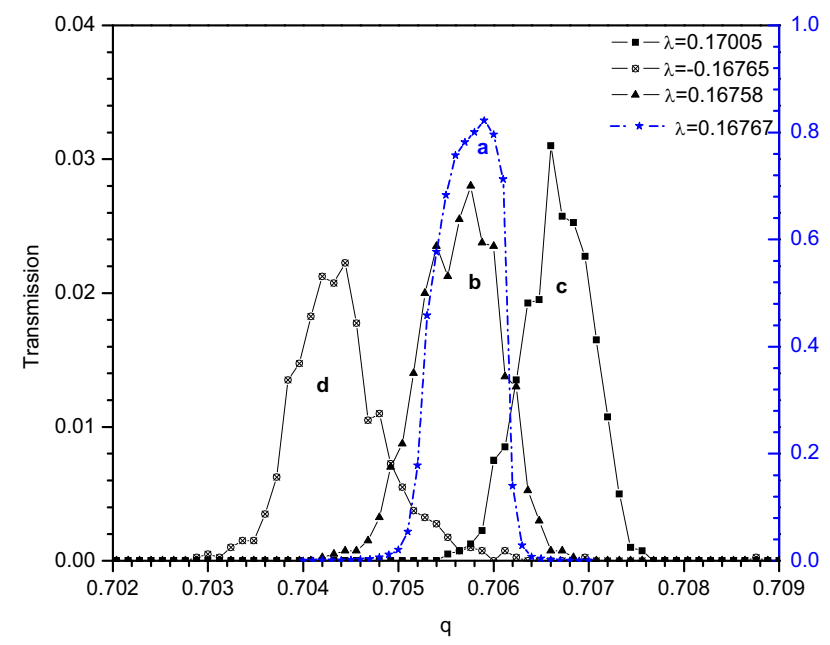

Figure 7. Peak shapes of (a) an ideal quadrupole without excitation, resolution 802 , (b) a round-rod quadrupole with $2.6 \%$ octopole field operated at the tip of the stability boundary without excitation $\left(q^{\prime}=0.00\right)$ with $a>0$, resolution $720,(c)$ a round-rod quadrupole with $2.6 \%$ octopole field operated at the tip of island A with greater $|a|\left(q^{\prime}=0.020\right.$ and $\left.v=9 / 10\right)$ with $a>0$, resolution $861,(d)$ a round rod quadrupole with $2.6 \%$ octopole field operated at the tip of island A with the lesser $|a|\left(q^{\prime}=0.020\right.$ and $\left.v=9 / 10\right)$ with $a<0$, resolution 782. The scale for the transmission of the ideal quadrupole is on the right. 
operation in island A with quadrupoles with 2 and $4 \%$ added octopole fields. Because the islands are essentially in the same position for these other values of $A_{4}$, scan lines with a given $\lambda$ give nearly the same resolution and transmission. With the negative DC applied to the smaller rods so that $a<0$, the added octopole leads to poor peak shape and resolution. Nevertheless, by operating at the tip of island $\mathrm{A}$ with the lesser $|a|$, mass analysis with resolution, transmission, and peak shape similar to that with $a>0$ is possible. Further work is required to determine if the use of islands generally can provide a method for mass analysis when added multipoles otherwise prevent mass analysis.

\section{Acknowledgments}

This work was supported by the Natural Sciences and Engineering Research Council of Canada and MDS SCIEX through an Industrial Research Chair.

\section{References}

1. Douglas, D. J.; Frank, A. J.; Mao, D. Linear Ion Traps in Mass Spectrometry. Mass Spec. Rev. 2005, 24, 1-29.

2. Schwartz, J. C.; Senko, M. W.; Syka, J. E. P. A Two-Dimensional Quadrupole Ion Trap Mass Spectrometer. J. Am. Soc. Mass Spectrom. 2002, 13, 659-669.

3. Hager, J. W. A New Linear Ion Trap Mass Spectrometer. Rapid Commun. Mass Spectrom. 2002, 16, 512-526.

4. (a) Campbell, J. M.; Collings, B. A.; Douglas, D. J. A New Linear Ion Trap Time-of-Flight System with Tandem Mass Spectrometry Capabilities. Rapid Commun. Mass Spectrom. 1998, 12, 1463-1474; (b) Collings, B. A.; Campbell, J. M.; Mao, D.; Douglas, D. J. A Combined Linear Ion Trap Time-of-Flight System with Improved Performance and MS Capabilities. Rapid Commun. Mass Spectrom. 2001, 15, 1777-1795.

5. Cha, B.; Blades, M.; Douglas, D. J. An Interface with a Linear Quadrupole Ion Guide for an Electrospray-Ion Trap Mass Spectrometer System. Anal. Chem. 2000, 72, 5647-5654

6. (a) Belov, M. E.; Anderson, G. A.; Angell, N. H.; Shen, Y.; Tolic, N.; Udseth, H. R.; Smith, R. D. Dynamic Range Expansion Applied to Mass Spectrometry Based on Data-Dependent Selective Ion Ejection in Capillary Liquid Chromatography FTICR for Enhanced Proteome Characterization. Anal. Chem. 2001, 73, 5052-5060; (b) Syka, J. E. P.; Marto, J. A.; Bai, D. L.; Horning, S.; Senko, M. W.; Schwartz, J. C.; Ueberheide, B.; Garcia, B.; Busby, S.; Muratore, T.; Shabanowitz, J.; Hunt, D. F. Novel Linear Quadrupole Ion Trap/FT Mass Spectrometer: Performance Characterization and Use in the Comparative Analysis of Histone $\mathrm{H}_{3}$ Post-Translational Modifications. J. Proteome Res. 2004, 3, 621-626.

7. (a) Hardman, M.; Makarov, A. Interfacing the Orbitrap Mass Analyzer to an Electrospray Ion Source. Anal. Chem. 2003, 75, 1699-1705; (b) Makarov, A.; Denisov, E.; Kholomeev, A.; Balschun, W.; Lange, O.; Strupat, K.; Horning, S. Performance Evaluation of a Hybrid Linear Ion Trap/Orbitrap Mass Spectrometer. Anal. Chem. 2006, 78, 2113-2120.
8. Franzen, J.; Gabling, R.-H.; Schubert, M.; Wang, Y. Nonlinear Ion Traps. In Practical Aspects of Ion Trap Mass Spectrometry; March R. E.; Todd J. F. J., Eds.; CRC Press: Boca Raton, 1995; Chap V1, pp 49-167.

9. (a) Michaud, A. L.; Frank, A. J.; Ding, C.; Zhao, X.; Douglas, D. J. Ion Excitation in a Linear Quadrupole Ion Trap with an Added Octopole Field. J. Am. Soc. Mass Spectrom. 2005, 16, 835-849; (b) Collings, B. A.; Stott, W. R.; Londry, F. A. Resonant Excitation in a Low Pressure Linear Ion Trap. J. Am. Soc. Mass Spectrom. 2003, 14, 622-634; (c) Collings, B. A. Increased Fragmentation Efficiency of Ions in a Low Pressure Linear Ion Trap with an Added dc Octopole Field. J. Am. Soc. Mass Spectrom. 2005, $16,1342-1352$.

10. (a) Sudakov, M. Effective Potential and the Ion Axial Beat Motion Near the Boundary of the First Stable Region in a Nonlinear Ion Trap. Int. J. Mass Spectrom. 2001, 206, 27-43; (b) Makarov, A. A. Resonance Ejection from the Paul Trap: A Theoretical Treatment Incorporating a Weak Octopole Field. Anal. Chem. 1996, 68, 4257-4263.

11. Sudakov, M.; Douglas, D. J. Linear Quadrupoles with Added Octopole Fields. Rapid Commun. Mass Spectrom. 2003, 17, 2290-2294.

12. (a) Konenkov, N.; Londry, F.; Ding, C.; Douglas, D. J. Linear Quadrupoles with Added Hexapole Fields. J. Am. Soc. Mass Spectrom. 2006, 17 1063-1073; (b) Wells, G. J. Linear Ion Trap Apparatus and Method Utilizing an Asymmetrical Trapping Field. US Patent 7034293, April 25, 2006.

13. Dawson P. H., Ed.; Quadrupole Mass Spectrometry and Its Applications; AIP Press: Woodbury, 1995; pp 19-21.

14. (a) Dawson, P. H.; Whetton, N. R. Nonlinear Resonances in Quadrupole Mass Spectrometers Due to Imperfect Fields. Int. J. Mass Spectrom. Ion Phys. 1969, 3, 1-12; (b) Dawson, P. H. Ion Optical Properties of Quadrupole Mass Filters. Adv. Electron., Electron. Optics. 1980, 53, 153-208.

15. Ding, C.; Konenkov, N. V.; Douglas, D. J. Quadrupole Mass Filters with Octopole Fields. Rapid Commun. Mass Spectrom. 2003, 17, 2495-2502.

16. (a) Hager, J. W. Performance Optimization and Fringing Field Modifications of a 24-mm Long RF-Only Quadrupole Mass Spectrometer. Rapid Commun. Mass Spectrom. 1999, 13, 740-748; (b) Londry, F. A.; Hager, J. W. Mass Selective Axial Ion Ejection from a Linear Quadrupole Ion Trap. J. Am. Soc. Mass Spectrom. 2003, 14, 1130-1147.

17. Moradian A.; Douglas, Donald J. High Scan Speed and Mass Resolution with Axial Ejection of Ions from Linear Quadrupolar Ion Traps with Added Octopole Fields. Proceedings of the 54th ASMS Conference on Mass Spectrometry and Allied Topics; Seattle, WA, May, 2006.

18. (a) Konenkov, N. V. Cousins, L. M. Baranov, V. I. Sudakov, M. Yu. Quadrupole Mass Filter Operation with Auxiliary Quadrupole Excitation: Theory and Experiment. Int. J. Mass Spectrom. 2001, 208, 17-27; (b) Baranov, V. I.; Konenkov, N. V.; Tanner, S. D. QMF Operation with Quadrupole Excitation; In Plasma Source Mass Spectrometry in the New Millennium; Holland, G; Tanner, S. D., Eds.; Royal Society of Chemistry: Cambridge, 2001; pp 63-72; (c) Konenkov, N. V.; Sudakov, M. Yu.; Douglas, D. J. Matrix Methods for the Calculation of Stability Diagrams in Quadrupole Mass Spectrometry. J. Am. Soc. Mass Spectrom. 2002, 13, 597-613.

19. Konenkov, N. V.; Korolkov, A. N.; Machmudov, M. Upper Stability Island of the Quadrupole Mass Filter with Amplitude Modulation of the Applied Voltages.J. Am. Soc. Mass Spectrom. 2005, 16, 379-387.

20. Sudakov, M.; Konenkov, N.; Douglas, D. J.; Glebova, T. Excitation Frequencies of Ions Confined in a Quadrupole Field with Quadrupole Excitation. I. Am. Soc. Mass Spectrom. 2000, 11, 10-18.

21. Douglas, D. J.; Glebova, T.; Konenkov, N.; Sudakov, M. Yu. Spatial Harmonics of the Field in a Quadrupole Mass Filter with Circular Electrodes. Technical Physics 1999, 44, 1215-1219.

22. Douglas, D. J.; Konenkov, N. V. Influence of the 6th and 10th Spatial Harmonics on the Peak Shape of a Quadrupole Mass Filter with Round Rods. Rapid Commun. Mass Spectrom. 2002, 16, 1425-1431.

23. Konenkov, N. V.; Douglas, D. J., unpublished. 